\title{
Multinuclear Spin Relaxation Study of Octaphenylcyclotetrasiloxane in Solution
}

\author{
Jozef Kowalewski ${ }^{\dagger}$ and Zulfiqar Ali \\ Division of Physical Chemistry, Arrhenius Laboratory, Stockholm University, S-106 91 Stockholm, Sweden
}

\begin{abstract}
Kowalewski, J. and Ali, Z., 1995. Multinuclear Spin Relaxation Study of Octaphenylcyclotetrasiloxane in Solution. - Acta Chem. Scand. 49: 734-736 () Acta Chemica Scandinavica 1995.

${ }^{13} \mathrm{C},{ }^{17} \mathrm{O}$ and ${ }^{29} \mathrm{Si}$ nuclear magnetic relaxation data are reported for octaphenylcyclotetrasiloxane in chloroform solution. The carbon-13 spin-lattice relaxation data are interpreted in terms of the Woessner model of internal rotational diffusion of the phenyl groups superimposed on the isotropic overall reorientation. The oxygen-17 spin-spin relaxation is assumed to be caused by the isotropic reorientation of the principal axis of the electric field gradient tensor. An oxygen-17 quadrupole coupling constant of $6.2 \pm 0.5 \mathrm{MHz}$ is obtained. The silicon-29 spinlattice relaxation is found to be dominated by dipole-dipole interaction with protons and to be very slow.
\end{abstract}

Some years ago, we reported a multinuclear $\left({ }^{13} \mathrm{C},{ }^{17} \mathrm{O}\right.$, $\left.{ }^{29} \mathrm{Si}\right)$ spin relaxation study of octamethylcyclotetrasiloxane (OMCTS) as a neat liquid. ${ }^{1}$ In this communication, we present a similar investigation of a related compound, octaphenylcyclotetrasiloxane (OPCTS) (1). OPCTS has

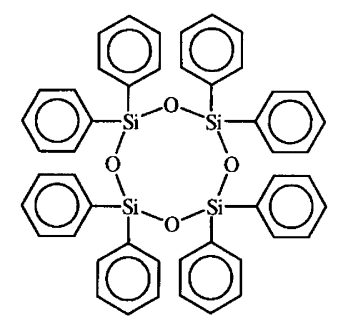

earlier been found to display an interesting phase behaviour (with a high-temperature mesophase occurring below the melting point at $204^{\circ} \mathrm{C}$ ); it has been studied by, among other methods, neutron diffraction ${ }^{2}$ and proton NMR spectroscopy. ${ }^{3}$ The compound is difficult to study in pure form by high-resolution NMR because of its high melting point. In this communication we report therefore an investigation of OPCTS in chloroform solution. We demonstrate that OPCTS is actually better suited for a multinuclear relaxation study than OMCTS [where it was necessary to combine the oxygen-17 relaxation data with quantum-chemical estimates of quadrupole coupling constant (QCC) in order to obtain the dynamic information] and that in this case it is possible to use carbon- 13 relaxation data to characterize the overall as well as the internal motion. By combining the carbon-13 results with

\footnotetext{
To whom correspondence should be addressed.
}

the oxygen-17 relaxation measurements, we estimate experimentally the oxygen-17 QCC in a siloxane chain. The procedure used is sometimes denoted as the "dual spin probe method' and has earlier been applied to determine oxygen-17 QCC in organic oxygen compounds. ${ }^{45}$ Measurements of the very slow silicon-29 spin-lattice relaxation are also reported.

\section{Experimental}

The octaphenylcyclotetrasiloxane $(97 \%)$ was obtained from Aldrich. The deuterated chloroform was puchased from Merck. Both compounds were used without further purification. The NMR sample was prepared as a $0.18 \mathrm{M}$ solution and transferred to a $10 \mathrm{~mm}$ o.d. NMR tube. The sample was degassed by several freeze-pump-thaw cycles and sealed under vacuum.

The ${ }^{13} \mathrm{C}$ and ${ }^{17} \mathrm{O}$ NMR experiments were performed on a Bruker MSL 200 spectrometer operating at $4.7 \mathrm{~T}$. The silicon-29 experiments were carried out on a $9.4 \mathrm{~T}$ Jeol Alpha 400 spectrometer. In both cases the standard broadband $10 \mathrm{~mm}$ probe was used, and the temperature was controlled using the standard variable-temperature accessories provided by the instrument manufacturers. The internal deuterium lock for field/frequency stabilization was used in all carbon and silicon experiments.

The ${ }^{13} \mathrm{C}$ and ${ }^{29} \mathrm{Si}$ measurements were carried out using WALTZ-16 proton decoupling with a $90^{\circ}$ proton pulse within the range $50-100 \mu \mathrm{s}$. The $90^{\circ}$ pulse width was 6.5 $\mu$ s for ${ }^{13} \mathrm{C}$ and $22 \mu \mathrm{s}$ for ${ }^{29} \mathrm{Si}$, the digital resolution was $1 \mathrm{~Hz}$ for both spin-1/2 nuclei, and the number of acquisitions was typically $8-16$. The spin-lattice relaxation time measurements were performed using the FIRFT method, ${ }^{6}$ and there were $8-12$ delays between the 180 
and $90^{\circ}$ pulses. The spin-lattice relaxation times $\left(T_{1}\right)$ were evaluated by using three-parameter least-squares fitting $^{7}$ of line intensities (obtained after an exponential broadening by $5-10 \mathrm{~Hz}$ ). The relaxation delay was about $1 \mathrm{~s}$ in the ${ }^{13} \mathrm{C}$ and $100 \mathrm{~s}$ in the ${ }^{29} \mathrm{Si}$ experiments. The accuracy of the $T_{1}$ measurements is estimated to be about $5 \%$, even though the standard deviations of the non-linear fits were better. The heteronuclear NOE factor was determined by taking a ratio, denoted $1+$ NOE, of exponentially broadened line intensities obtained using the dynamic NOE sequence ${ }^{8}$ with a long $\left(>5 T_{1}\right)$ and a short $(10 \mathrm{~ms})$ proton irradiation before the observe pulse. The ralaxation delay was $(8-10) T_{1}$ in all NOE experiments. The accuracy of the intensity ratio is of the order of $5-10 \%$. All the experiments were performed at least twice, and the mean values are reported.

The $90^{\circ}$ pulse width in the ${ }^{17} \mathrm{O}$ experiments was $11 \mu \mathrm{s}$, the digital resolution was $25 \mathrm{~Hz}$, and the number of scans was typically about $(1-2) \times 10^{6}$. The spin-spin relaxation time was determined from the linewidth by means of the relationship $T_{2}{ }^{-1}=\pi \Delta v$. The linewidth $\Delta v$ was evaluated by fitting the spectra to a single Lorentzian using the program FELIX (from Biosym Technologies) after transferring the data to a SUN Sparc workstation. The uncertainty of the linewidth determination is of the order of $5-10 \%$.

\section{Results and discussion}

Carbon-13 relaxation. The spin relaxation of carbon- 13 nuclei in $\mathrm{CH}$ groups is usually dominated by the dipoledipole (DD) interaction with the magnetic moments of directly bonded protons. Under proton decoupling, the relaxation is single exponential with a time constant denoted $T_{1}$. Under extreme narrowing conditions, the spinlattice relaxation rate due to the DD interaction can be obtained by a combination of measurements of $T_{1}$ and the NOE factor. It is given by ${ }^{9,10}$

$T_{\mathrm{1DD}}^{-1}=T_{\text {lobs }}^{-1} \frac{\mathrm{NOE}_{\mathrm{obs}}}{\left(\gamma_{\mathrm{H}} / 2 \gamma_{\mathrm{C}}\right)}=\left(\frac{\mu_{0}}{4 \pi}\right)^{2}\left(\frac{\gamma_{\mathrm{C}}^{2} \gamma_{\mathrm{H}}^{2} h^{2}}{r_{\mathrm{CH}}^{6}}\right) \tau_{\mathrm{c}}^{\text {eff }}$

$T_{\text {lobs }}{ }^{-1}$ is the measured relaxation rate, $\mathrm{NOE}_{\mathrm{obs}}$ is the observed nuclear Overhauser enhancement and $\left(\gamma_{\mathrm{H}} / 2 \gamma_{\mathrm{C}}\right)$ is the largest possible value of the carbon-13 NOE, equal to 1.99. $\mu_{0}$ is the permeability of vacuum, $\gamma_{C}$ and $\gamma_{H}$ are the magnetogyric ratios for carbon-13 and proton, respec- tively, $r_{\mathrm{CH}}$ is the internuclear distance and $\tau_{\mathrm{c}}^{\text {eff }}$ is the effective correlation time for the reorientation of the carbon-proton internuclear axis.

The effective correlation time in eqn. (1) can be expressed in terms of more fundamental quantities, if a specified dynamic model is chosen. It is worth stressing that the concept of effective correlation time is meaningful only if extreme narrowing conditions $\left(\tau_{\mathrm{c}}{ }^{2} \omega_{i}{ }^{2} \ll 1\right)$ apply to all relevant motions, characterized by the correlation times $\tau_{c}$, and all relevant angular frequencies $\omega_{i}$. The simplest possible dynamic model assumes that the molecular axis under consideration reorients according to the isotropic rotational diffusion of a rigid body, which gives $\tau_{\mathrm{c}}{ }^{\text {eff }}=1 / 6 D_{\mathrm{R}}$, where $D_{\mathrm{R}}$ is the rotational diffusion constant. This model is unlikely to be valid for the motion of the $\mathrm{CH}$ vectors in OPCTS, as the phenyl rings can be expected to undergo internal reorientations independent of the overall rotation of the molecule. A simple dynamic model describing a superposition of isotropic overall reorientation and interval rotation, with the two processes described by the diffusion constants $D_{\mathrm{R}}$ and $D_{\mathrm{i}}$, respectively, has been formulated by Woessner. ${ }^{11}$ The effective correlation time is in this model expressed in terms of these two diffusion coefficients (or two correlation times) and geometrical parameters determined by angle $\theta$ between the $\mathrm{CH}$ axis and the internal rotation axis. ${ }^{9-11}$

We assume that the internal rotation axis of the phenyl group coincides with the silicon-to- $\mathrm{C}_{\mathrm{ipso}}$ bond axis, which in turn coindices with the $\mathrm{C}_{\text {para }} \mathrm{H}$ bond. Moreover, we assume that the phenyl rings have an idealized geometry, with all bond angles equal to $120^{\circ}$. With these assumptions, the angle $\theta$ is 0 for the $\mathrm{C}_{\text {para }} \mathrm{H}$ axis and $60^{\circ}$ for the $\mathrm{C}_{\text {metu }} \mathrm{H}$ and $\mathrm{C}_{\text {ortho }} \mathrm{H}$ axes. This means that the para carbon relaxation is insensitive to the internal motion and can directly yield information on the overall reorientation. The relaxation of the meta and ortho carbons can then be used to estimate $D_{\mathrm{i}}$. The relaxation properties of the two carbons are expected to be identical.

The four carbon signals of OPCTS (consistent with the equivalence of the eight phenyl rings) can easily be assigned on the basis of intensity (the two more intense signals are ortho and meta) and relaxation rates $\left(\mathrm{C}_{\text {para }}\right.$ relaxes much faster than $\mathrm{C}_{i p s o}$, which does not carry any protons). In fact, one of the ortho or meta carbon signals overlaps with that of $\mathrm{C}_{i p s o}$; in the following we concentrate on the relaxation of the non-overlapping signal, denoted $\mathrm{C}_{o, m}$. The relaxation rates and NOEs of the two carbons of interest, measured at different temperatures, are summarized in Table 1, and the derived dynamic

Table 1. Relaxation data for ${ }^{13} \mathrm{C}$ in OPCTS.

\begin{tabular}{|c|c|c|c|c|c|c|}
\hline \multirow[b]{2}{*}{$T / K$} & \multicolumn{3}{|l|}{$\mathrm{C}_{\text {para }}$} & \multicolumn{3}{|l|}{$\mathrm{C}_{o, m}$} \\
\hline & $T_{1 \mathrm{obs}}{ }^{-1} / \mathrm{s}^{-1}$ & $\mathrm{NOE}_{\mathrm{obs}}$ & $T_{1 \mathrm{DD}}-1 / \mathrm{s}^{-1}$ & $T_{1 \mathrm{obs}}{ }^{-1} / \mathrm{s}^{-1}$ & $\mathrm{NOE}_{\mathrm{obs}}$ & $T_{1 \mathrm{DD}}{ }^{-1 / \mathrm{s}^{-1}}$ \\
\hline 286 & 2.44 & 1.90 & $2.3 \pm 0.2$ & 0.60 & 1.99 & $0.60 \pm 0.06$ \\
\hline 303 & 1.89 & 1.87 & $1.8 \pm 0.2$ & 0.46 & 1.91 & $0.44 \pm 0.04$ \\
\hline 323 & 1.47 & 1.89 & $1.40 \pm \overline{0} .14$ & 0.34 & 1.82 & $0.31 \pm 0.03$ \\
\hline
\end{tabular}


Table 2. Molecular dynamics data from carbon-13 relaxation in OPCTS.

\begin{tabular}{lclll}
\hline$T / \mathrm{K}$ & $\tau_{\mathrm{c}}{ }^{\mathrm{eff}}\left(\mathrm{C}_{\text {para }}\right) / \mathrm{ps}$ & $D_{\mathrm{R}} / 10^{9} \mathrm{~s}^{-1}$ & $\tau_{\mathrm{c}}{ }^{\mathrm{eff}}\left(\mathrm{C}_{o, m}\right) / \mathrm{ps}$ & $D_{\mathrm{i}} / 10^{9} \mathrm{~s}^{-1}$ \\
\hline 286 & $109 \pm 16$ & $1.5 \pm 0.2$ & $28 \pm 4$ & $18 \pm 4$ \\
303 & $82 \pm 12$ & $2.0 \pm 0.3$ & $20 \pm 3$ & $25 \pm 5$ \\
323 & $65 \pm 10$ & $2.6 \pm 0.4$ & $14 \pm 2$ & $37 \pm 7$ \\
\hline
\end{tabular}

quantities are listed in Table 2. Estimated uncertainties in all derived quantities are also listed in the tables.

The temperature dependence of the rotational diffusion constants $D_{\mathrm{R}}$ and $D_{\mathrm{i}}$ is commonly interpreted in terms of Arrhenius-type equation. ${ }^{10}$ The activation energy for $D_{\mathrm{R}}$ derived in this way is about $11 \mathrm{~kJ} \mathrm{~mol}^{-1}$, while the activation energy of $D_{\mathrm{i}}$ is about $15 \mathrm{~kJ} \mathrm{~mol}^{-1}$.

Oxygen-17 and silicon-29 relaxation. The oxygen-17 signal of OPCTS is a single, fairly broad line, corresponding to all oxygen atoms being magnetically equivalent. The linewidths and derived quantities (including estimated uncertainties) are presented in Table 3 . Under extreme narrowing conditions, the $I=5 / 2$ oxygen- 17 nuclei relax exponentially with the spin-lattice relaxation rate equal to the spin-spin relaxation rate, given by 9.10 .12

$T_{2 Q}^{-1}=\frac{3 \pi^{2}}{10}\left[\frac{2 I+3}{I^{2}(2 I-1)}\right]\left(1+\frac{\varepsilon^{2}}{3}\right)\left(\frac{e^{2} q Q}{h}\right)^{2} \tau_{c}^{e f f}$

$Q$ is the nuclear electric quadrupole moment and $q$ is the principal component of the field gradient tensor at the site of the nucleus; $\left(e^{2} Q q / h\right)$ is called the quadrupole coupling constant, QCC. $\varepsilon$ is the asymmetry parameter of the field gradient tensor at the nuclear site. QCC and $\varepsilon$ are related to the electronic structure around the oxygen atom and can therefore carry structural information. The effective rotational correlation time $\tau_{c}^{\text {eff }}$ in eqn. (2) refers to the reorientation of the principal axis of the electric field gradient tensor. If the overall reorientation of the molecule is isotropic and if the cyclotetrasiloxane ring is rigid, then the correlation times for the reorientation of the $\mathrm{C}_{p a r a} \mathrm{H}$, in eqn. (1), and of the principal axis of the field gradient tensor at the oxygen atom, in eqn. (2), are identical. The correlation times for the para carbon from Table 2 can then be used, together with the oxygen- 17 relaxation rates, to estimate the product of the square of QCC and the factor $\left(1+\varepsilon^{2} / 3\right)$. For not too large asymmetry parameters, the latter factor does not differ much from unity. The asymmetry parameter for the oxygen-17 in OMCTS, obtained form $a b$ initio calculations, was about $0.2-0.3{ }^{1}$

Table 3. Relaxation data for ${ }^{17} \mathrm{O}$ in OPCTS.

\begin{tabular}{lllll}
\hline$T / \mathrm{K}$ & Linewidth $/ \mathrm{Hz}$ & $T_{2}{ }^{-1} / 10^{3} \mathrm{~s}^{-1}$ & $\tau_{\mathrm{c}}{ }^{\text {eff }} / \mathrm{ps}^{a}$ & $\mathrm{OCC} / \mathrm{MHz}$ \\
\hline 286 & 1130 & $3.5 \pm 0.3$ & $109 \pm 16$ & $5.9 \pm 0.7$ \\
303 & 1020 & $3.2 \pm 0.3$ & $82 \pm 12$ & $6.4 \pm 0.8$ \\
323 & 760 & $2.4 \pm 0.2$ & $65 \pm 10$ & $6.2 \pm 0.8$ \\
\hline
\end{tabular}

${ }^{a}$ From Table 2.
At the level of accuracy of our determination of the rotational correlation time, the correction of $1-3 \%$ due to an asymmetry parameter of this magnitude can be neglected, and the oxygen-17 relaxation rates can directly be used to estimate the oxygen QCC as a function of temperature, cf. Table 3 . We can see that the three values agree with each other, and we propose a mean value of $6.2 \mathrm{MHz}$ as the value of the QCC, with an uncertainty of about $\pm 0.5 \mathrm{MHz}$. This agrees very well with the estimates of 6.3-6.4 MHz for OMCTS, ${ }^{1}$ based on scaled $a b$ initio quantum chemistry results.

Because of the magnetic equivalence of the phenyl rings and oxygen atoms, the same can be expected for silicon. Indeed, the silicon-29 spectrum of OPCTS consists of a single, narrow line, characterized by very slow spin-lattice relaxation. A relaxation time of about $120 \mathrm{~s}$ was obtained at $30^{\circ} \mathrm{C}$. According to the NOE measurement, which yielded NOE of about -2 (the largest possible NOE for silicon is -2.52 ), the dipole-dipole interaction is the most important relaxation mechanism. Since there are no protons derectly bonded to the silicon, the quantitative interpretation of the silicon relaxation is complicated. Thus the value of the time-consuming silicon-29 relaxation measurements in OPCTS is limited, in full analogy with the case of OMCTS, and we chose not to repeat the silicon measurements at other temperatures.

In conclusion, we have demonstrated that the carbon-13 relaxation in the phenyl groups of OPCTS carries information on the overall as well as internal reorientational dynamics in solution. This information can be used, in connection with oxygen-17 relaxation, to provide quadrupole coupling constant data of potential structural relevance.

Acknowledgements. This work has been supported by the Swedish Natural Science Research Council.

\section{References}

1. Kowalewski, J. and Berggren, E. Magn. Reson. Chem. 27 (1989) 386.

2. Volino, F. and Dianoux, A. J. Ann. Phys. 3 (1978) 151.

3. Moore, T. C., McCormick, W. D. and Wade, C. G. J. Phys. Chem. 89 (1985) 3936.

4. Larsson, K. and Kowalewski, J. Acta Chem. Scand., Ser. A40 (1984) 218.

5. Delseth, C. and Kintzinger, J.-P. Helv. Chim. Acta 65 (1982) 2273.

6. Canet, D., Levy, G. C. and Peat, I. R. J. Magn. Reson. 18 (1975) 199.

7. Kowalewski, J., Levy, G. C., Johnson, L. F. and Palmer, L. J. Magn. Reson. 26 (1977) 533.

8. Kowalewski, J., Ericsson, A. and Vestin, R. J. Magn. Reson. 31 (1978) 165.

9. Harris, R. K., Nuclear Magnetic Resonance Spectroscopy, Longman, Harlow 1986.

10. Kowalewski, J. Ann. Rep. NMR Spectrosc. 22 (1990) 307; 23 (1991) 289.

11. Woessner, D. E. J. Chem. Phys. 36 (1962) 1.

12. Abragam, A. The Principles of Nuclear Magnetism, Oxford University Press, Oxford 1961.

Received February 8, 1995. 